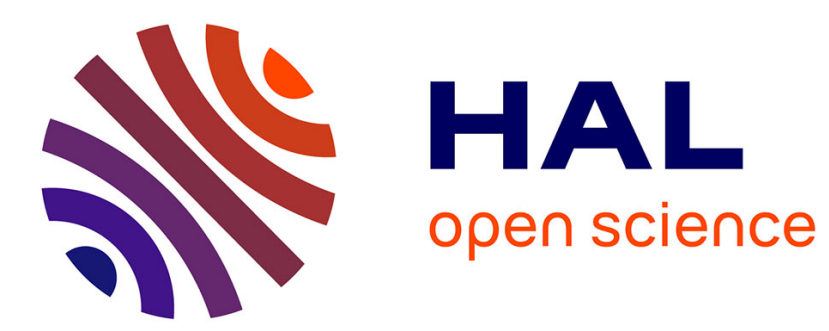

\title{
A combinatorial approach to jumping particles II: general boundary conditions
}

\author{
Enrica Duchi, Gilles Schaeffer
}

\section{To cite this version:}

Enrica Duchi, Gilles Schaeffer. A combinatorial approach to jumping particles II: general boundary conditions. 2004, pp.12. hal-00159627

\section{HAL Id: hal-00159627 \\ https://hal.science/hal-00159627}

Submitted on 6 Jul 2007

HAL is a multi-disciplinary open access archive for the deposit and dissemination of scientific research documents, whether they are published or not. The documents may come from teaching and research institutions in France or abroad, or from public or private research centers.
L'archive ouverte pluridisciplinaire HAL, est destinée au dépôt et à la diffusion de documents scientifiques de niveau recherche, publiés ou non, émanant des établissements d'enseignement et de recherche français ou étrangers, des laboratoires publics ou privés. 


\title{
A combinatorial approach to jumping particles II: general boundary conditions
}

\author{
Enrica Duchi and Gilles Schaeffer
}

\begin{abstract}
We consider a model of particles jumping on a row of cells, called in physics the one dimensional totally asymmetric exclusion process with open boundaries (TASEP). From the point of view of combinatorics a remarkable feature of this Markov chain is that Catalan numbers are involved in several entries of its stationary distribution.

In a companion paper, we gave a combinatorial interpretation and a simple proof of these observations in the simplest case where the particles enter, jump and exit at the same rate. To do this we revealed a second row in which particles travel backward and defined on these two row configurations a Markov chain with uniform stationary distribution which is a covering of the TASEP.

In this paper we show how to deal with general rates. The covering chain is still defined on two row configurations, but its stationary distribution is not uniform anymore. Instead it is described in terms of two natural combinatorial statistics.
\end{abstract}

\section{Jumping particles}

We shall consider a model of jumping particles on a row of $n$ cells that was studied by and large since the early 90 's in physics, under the name one dimensional totally asymmetric exclusion process with boundaries, or TASEP for short. Roughly speaking, the TASEP consists of black particles entering a row of $n$ cells from an infinite reservoir on the left hand side and randomly hopping to the right with the simple exclusion rule that each cell may contain only one particle.

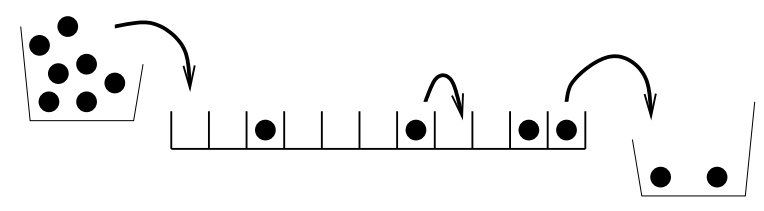

Figure 1: An informal illustration of the TASEP.

The TASEP is usually defined as a continuous-time Markov process on a finite set of configurations of particles on a line. We shall use an alternative definition as a finite state Markov chain - with discrete time- which is more convenient for our combinatorial purpose. One could insist on calling our chain the TASEC, with "C" for chain instead of "P" for process, but as we will argue later, there is no need for this distinction. Another cosmetic modification we allow ourselves consists in putting a white particle in each empty cell, to make explicit the leftright particle-hole symmetry of the system. 


\subsection{The basic system, or TASEP}

A basic configuration is a row of $n$ cells, each containing either one black particle or one white particle (see Figure 2). These cells are delimited by $n+1$ walls: the left border (or wall 0 ), the $i$ th separation wall for $i=1, \ldots, n-1$, and the right border (or wall $n$ ).

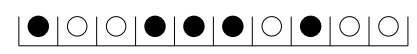

Figure 2: A basic configuration with $n=10$ cells.

The TASEP, which we shall sometimes refer to as the basic system, is a Markov chain $S_{\alpha \beta \gamma}$ defined for any three parameters $\alpha, \beta$ and $\gamma$ in the interval $[0,1]$ on the set of basic configurations. From time $t$ to $t+1$, the chain evolves from the basic configuration $\tau=S_{\alpha \beta \gamma}(t)$ to a basic configuration $\tau^{\prime}=S_{\alpha \beta \gamma}(t+1)$ as follows:

- A wall $i$ is chosen uniformly at random among the $n+1$ walls, and then may become active with probability $\lambda(i)$, with $\lambda(i)=\alpha$ for $i=1, \ldots, n-1$, $\lambda(0)=\beta$ and $\lambda(n)=\gamma$.

- If the wall did not become active, then nothing happens: $\tau^{\prime}=\tau$.

- Otherwise the configuration may change near the active wall:

$a$. If the active wall is not on the border $(i \in\{1, \ldots, n-1\})$ and has a black particle on its left and a white one on its right, then these two particles swap: $\bullet \mid \circ \rightarrow$ ○|•.

$b$. If the active wall is the left border $(i=0)$ and the leftmost cell contains a white particle, then the white particle leaves the system and it is replaced by a black particle: $|\circ \rightarrow| \bullet$.

$c$. If the active wall is the right border $(i=n)$ and the rightmost cell contains a black particle, then the black particle leaves the system and it is replaced by a white particle: $\bullet \mid \rightarrow$ o|.

d. Otherwise the configuration is left unchanged.

The four cases $a, b, c, d$ define an application $\vartheta$ from the set of configurations with an active wall into the set of configurations, and, in terms of this application, the chain can be described as: let $I(t)$ be a sequence of independent uniform random variables on $\{0, \ldots, n\}$, and set

$$
S_{\alpha \beta \gamma}(t+1)=\left\{\begin{array}{l}
\vartheta\left(S_{\alpha \beta \gamma}(t), I(t)\right) \text { with probability } \lambda(I(t)) \\
S_{\alpha \beta \gamma}(t) \text { otherwise, }
\end{array}\right.
$$

where $\lambda(i)=\alpha$ for $i \in\{1, \ldots, n-1\}, \lambda(0)=\beta$, and $\lambda(n)=\gamma$.

As illustrated by Figure 3, during the evolution of the system, black particles travel from left to right while white particles do the opposite. As already mentioned, one can equivalently think about white particles as empty cells. See Figure 10 for the entire system with $n=3$. 


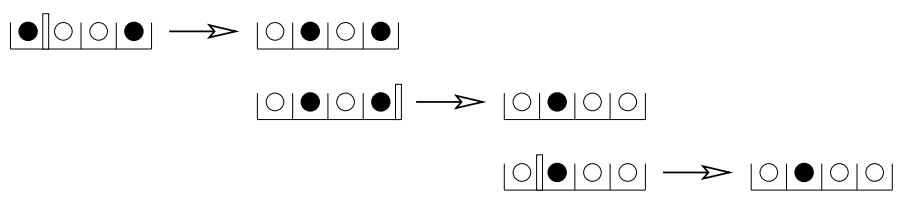

Figure 3: A possible evolution, with $n=4$. The active wall triggering each transition is indicated.

\subsection{A remarkable stationary distribution}

Among many results on the TASEP, Derrida et al. $[1,3]$ proved the following nice property of the system in the case $\alpha=\beta=\gamma=1$. First,

$$
\operatorname{Prob}\left(S_{111}(t) \text { contains } 0 \text { black particles) } \underset{t \rightarrow \infty}{\longrightarrow} \frac{1}{C_{n+1}},\right.
$$

where $C_{n+1}=\frac{1}{n+2}\left(\begin{array}{c}2 n+2 \\ n+1\end{array}\right)$ is the $(n+1)$ th Catalan number. More generally, for all $0 \leq k \leq n$,

$$
\operatorname{Prob}\left(S_{111}(t) \text { contains } k \text { black particles }\right) \underset{t \rightarrow \infty}{\longrightarrow} \frac{\frac{1}{n+1}\left(\begin{array}{c}
n+1 \\
k
\end{array}\right)\left(\begin{array}{c}
n+1 \\
n-k
\end{array}\right)}{C_{n+1}} .
$$

The finite state Markov chain $S_{111}$ is clearly ergodic so that the previous limits are in fact the probabilities of the same events in the unique stationary distribution of the chain [6]. More generally, Derrida et al. provided expressions for the stationary probabilities of $S_{\alpha \beta \gamma}$. Since their original work a number of papers have appeared, providing alternative proofs and further results on correlations, time evolutions, etc. It should be moreover stressed that the model we presented is a special case among the many existing variants of asymmetric exclusion processes. See for instance the article [4] for recent advances and a bibliography. However, the remarkable apparition of Catalan numbers is not easily understood from the proofs in the physics literature. As far as we know, these proofs rely either on a matrix ansatz, or on a Bethe ansatz, both being then proved by a recursion on $n$.

In a previous paper [5], we proposed a combinatorial proof of Formulas (1) and (2) based on a combinatorial interpretation of the stationary distribution of $S_{111}$. The aim of the present paper is to give a combinatorial derivation of the general stationary distribution of $S_{\alpha \beta \gamma}$.

\subsection{The complete system}

The main ingredient we introduced in [5] to study the TASEP consisted in a new Markov chain $X_{111}$ on a set $\Omega_{n}^{0}$ of complete configurations, that satisfies two main requirements. On the one the stationary distribution of the basic chain $S_{111}$ can be simply expressed in terms of that of the chain $X_{111}$. On the other hand the stationary behavior of the chain $X_{111}$ is easy to understand. The complete configurations that we introduced for this purpose are made of two rows of $n$ cells containing black and white particles. The first requirement was met by imposing that in the first row, the chain $X_{111}$ simulates the chain $S_{111}$, i.e. $X_{111}$ is a covering chain of $S_{111}$. The second requirement was met by adequately choosing the complete configurations and the transition rules so that $X_{111}$ has clearly a uniform stationary distribution. In this paper we shall proceed in an analogous way 


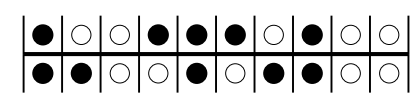

Figure 4: A complete configuration with $n=10$.

and construct a complete chain $X_{\alpha \beta \gamma}$ on $\Omega_{n}^{0}$, that will allow us to describe the stationary distribution of the basic chain $S_{\alpha \beta \gamma}$.

A complete configuration of $\Omega_{n}^{0}$ is a pair of rows of $n$ cells satisfying the following constraints:

(i) The balance condition: The two rows contain together $n$ black and $n$ white particles.

(ii) The positivity condition: On the left hand side of any vertical wall there are no more white particles than black ones.

An example of complete configuration is given in Figure 4. In view of Formulas (1) and (2), one first reason to introduce these complete configurations is that the cardinal of $\Omega_{n}^{0}$ is $\frac{1}{n+2}\left(\begin{array}{c}2 n+2 \\ n+1\end{array}\right)$, and that, for all $0 \leq k \leq n$, the cardinal of the set $\Omega_{k, m}^{0}$ of complete configurations with $k$ black and $m=n-k$ white particles on the top row is $\frac{1}{n+1}\left(\begin{array}{c}n+1 \\ k\end{array}\right)\left(\begin{array}{c}n+1 \\ n-k\end{array}\right)$. These formulas can be obtained in many ways, for instance using the cycle lemma (see [5]), or through one-to-one correspondences between complete configurations and bicolored Motzkin paths with $n$ steps, or Dyck paths with $2 n+2$ steps [8, Chap. 6 ]. Yet another classical way to obtain them is using generating functions, as we shall do in Section 2.

The Markov chain $X_{\alpha \beta \gamma}$ on $\Omega_{n}^{0}$ will be defined in terms of an application $T$ from the set $\Omega_{n}^{0} \times\{0, \ldots, n\}$ to the set $\Omega_{n}^{0}$. This application, which we already used in [5], is derived in Section 3 as the first component of a fundamental bijection $\bar{T}$ and can be conveniently described as follows. Given a complete configuration $\omega$ and an active wall $i$, the actions of $T$ on the first row of $\omega$ do not depend on the second row, and mimic the application $\vartheta$ describing the evolution of the Markov chain $S_{\alpha \beta \gamma}$ in the cases $a, b, c$ and $d$ of the description of the basic TASEP. In particular in the top row, black particles travel from left to right and white particles from right to left. As opposed to that, in the bottom row, $T$ moves black and white particles backward. In order to describe this, we first introduce the concept of sweep (see Figure 5):

- A white sweep between walls $i_{1}$ and $i_{2}$ consists in all white particles of the bottom row and between walls $i_{1}$ and $i_{2}$ simultaneously hopping to the right (some black particles thus being displaced to the left in order to fill the gaps).

- A black sweep between walls $i_{1}$ and $i_{2}$ consists in all black particles of the bottom row and between walls $i_{1}$ and $i_{2}$ simultaneously hopping to the left (some white particles thus being displaced to the right in order to fill the gaps).

Next, around the active wall $i$, we distinguish the following walls: if $i \neq 0$, let $j_{1}<i$ the leftmost wall such that there are only white particles in the top row between walls $j_{1}$ and $i-1$; if $i \neq n$, let $j_{2}>i$ be the rightmost wall such that there are only black particles in the bottom row between walls $i+1$ and $j_{2}$. 

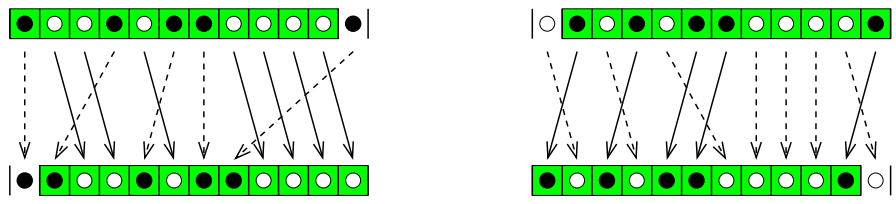

Figure 5: A white sweep and a black sweep.

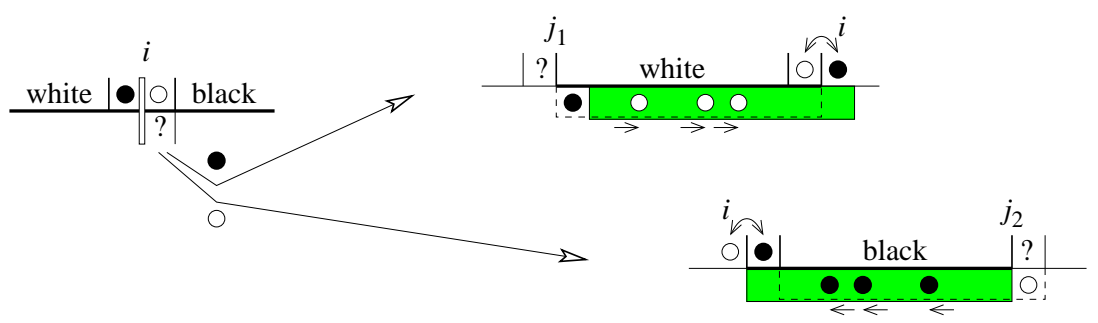

Figure 6: Sweeps occurring below the transition $(b|w \rightarrow w| b)$.

With these definitions, we are in position to describe completely the application $T$. Given a configuration $\omega \in \Omega_{n}^{0}$ and an active wall $i \in\{0, \ldots, n\}$, the cases $a, b c$ and $d$ of the basic chain describe the first row of $T(\omega, i)$, and they are complemented as follows to give the second row:

$a$. Depending whether the particle on the bottom right of the $i$ th wall in $\omega$ is black or white, a white sweep occurs between $j_{1}$ and $i$, or a black one between $i+1$ and $j_{2}+1$ (see Figure 6).

$b$. The leftmost column of $\omega$ consists of a $\left.\right|_{.} ^{\circ} \mid$-column. These two particles exchange (in agreement with the rule for the top row), and a black sweep occurs between the left border and wall $j_{2}+1$.

c. The rightmost column of $\omega$ consists of a $\left.\right|_{0} ^{\bullet} \mid$-column. These two particles exchange (in agreement with the rule for the top row), and a white sweep occurs between wall $j_{1}$ and the right border.

$d$. As in the top row, nothing happens in the bottom row.

The Markov chain $X_{\alpha \beta \gamma}$ is the Markov chain on the set $\Omega_{n}^{0}$ of complete configurations that is defined from the application $T$ exactly as the TASEP is described from $\vartheta$ : the evolution rule from time $t$ to $t+1$ consists in choosing $i=I(t)$ uniformly at random in $\{0, \ldots, n\}$ and setting

$$
X_{\alpha \beta \gamma}(t+1)=\left\{\begin{array}{l}
T\left(X_{\alpha \beta \gamma}(t), i\right) \text { with probability } \lambda(i), \\
X_{\alpha \beta \gamma}(t) \text { otherwise, }
\end{array}\right.
$$

where $\lambda(i)=\alpha$ for $i \in\{1, \ldots, n-1\}, \lambda(0)=\beta$, and $\lambda(n)=\gamma$.

By construction, the Markov chains $S_{\alpha \beta \gamma}$ and $X_{\alpha \beta \gamma}$ are related by

$$
S_{\alpha \beta \gamma} \equiv \operatorname{top}\left(X_{\alpha \beta \gamma}\right)
$$




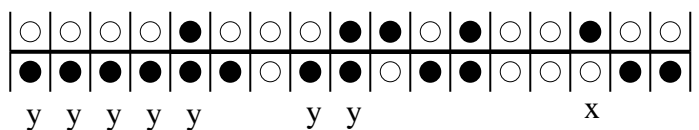

Figure 7: A configuration $\omega$ with weight $q(\omega)=\alpha^{8} \beta^{10} \gamma^{16}$. Labels are indicated below particles.

where $\operatorname{top}(\omega)$ denotes the top row of a complete configuration $\omega$, and the $\equiv$ is intended as identity in law. An appealing interpretation from a combinatorial point of view is that we have revealed a circulation of the particles, that use the bottom row to travel backward and implement the infinite reservoirs.

\subsection{The stationary distribution of the complete system}

In order to express the stationary distribution of the chain $X_{\alpha \beta \gamma}$, we introduce two combinatorial statistics and use them to associate a weight $q(\omega)$ to each complete configuration.

By definition, a complete configuration $\omega$ is a concatenation of four types of columns $\left.\left.\right|_{\bullet} ^{\bullet}||\right|_{0} ^{,\bullet}|,|_{0}^{\circ} \mid$ and $\left.\right|_{0} ^{\circ} \mid$, subject to the balance and positivity conditions. Observe that the concatenation of two complete configurations of $\Omega_{i}^{0}$ and $\Omega_{j}^{0}$ with $i+j=n$ yields a complete configuration of $\Omega_{n}^{0}$. Let us call prime a configuration that cannot be decomposed in this way. A complete configuration $\omega$ can be uniquely written as a concatenation $\omega=\omega_{1} \cdots \omega_{m}$ of prime configurations. These prime factors can be of three types: $|:|$-columns, $\left.\right|_{0} ^{0} \mid$-columns, and blocks of the form $\left.|:| \omega^{\prime}\right|_{0} ^{0} \mid$ with $\omega^{\prime}$ a complete configuration. The inner part $\omega^{\prime}$ of a block $\omega=\left.|:| \omega^{\prime}\right|_{0} ^{0} \mid$ is referred to as its inside.

Now, given a complete configuration $\omega$, let us assign labels to some of the black and white particles of its bottom row: a white particle is labeled $x$ if it is not in a block, and a black particle is labeled $y$ if it is not in the inside of a block and if on its left hand side, all white particles belong to some block. Let us denote by $n_{x}(\omega)$ and $n_{y}(\omega)$ respectively the number of labels of type $x$ and the number of labels of type $y$ in the configuration $\omega$. Then the weight of a configuration $\omega$ is defined as

$$
q(\omega)=\beta^{n} \gamma^{n}\left(\frac{\alpha}{\beta}\right)^{n_{y}(\omega)}\left(\frac{\alpha}{\gamma}\right)^{n_{x}(\omega)}=\alpha^{n_{x}(\omega)+n_{y}(\omega)} \beta^{n-n_{y}(\omega)} \gamma^{n-n_{x}(\omega)} .
$$

For instance, the weight of the configuration of Figure 7 is $\alpha^{8} \beta^{10} \gamma^{16}$, and more generally the weight is a monomial with total degree $2 n$.

Theorem 1.1 The Markov chain $X_{\alpha \beta \gamma}$ is ergodic and has the following stationary distribution:

$$
\operatorname{Prob}\left(X_{\alpha \beta \gamma}(t)=\omega\right) \underset{t \rightarrow \infty}{\longrightarrow} \frac{q(\omega)}{Z_{n}}, \quad \text { where } Z_{n}=\sum_{\omega^{\prime} \in \Omega_{n}^{0}} q\left(\omega^{\prime}\right),
$$

where $q(\omega)$ is the previously defined weight on complete configurations.

In particular for $\alpha=\beta=\gamma=1, q(\omega)=1$ for all configurations and we recover uniformity as in [5],

$$
\operatorname{Prob}\left(X_{111}(t)=\omega\right) \underset{t \rightarrow \infty}{\longrightarrow} \frac{1}{\left|\Omega_{n}^{0}\right|}=\frac{1}{C_{n}} .
$$


The Markov chain $X_{\alpha \beta \gamma}$ is clearly aperiodic, and the fact that it is irreducible follows from the irreducibility of $S_{111}$ done in [5]. This granted, it is sufficient for the proof of Theorem 1.1 to show that the distribution induced by the weights $q$ is stationary. We shall use an alternate description of $T$, which rely on the following result proved in Section 3.

Theorem 1.2 There exists a bijection $\bar{T}$ from $\Omega_{n}^{0} \times\{0, \ldots, n\}$ onto itself such that

- the application $T$ is the first component of $\bar{T}$ : for all $\omega$ and $i, \bar{T}(\omega, i)=$ $\left(\omega^{\prime}, j\right) \Rightarrow T(\omega, i)=\omega^{\prime}$,

- the bijection $\bar{T}$ transports weights: for all $\left(\omega^{\prime}, j\right)=\bar{T}(\omega, i)$,

$$
\lambda(j) q\left(\omega^{\prime}\right)=\lambda(i) q(\omega),
$$

where $\lambda(i)=\alpha$ for $i \in\{1, \ldots, n-1\}, \lambda(0)=\beta$ and $\lambda(n)=\gamma$.

In order to see that the distribution induced by $q$ is stationary, we assume that

$$
\operatorname{Prob}\left(X_{\alpha \beta \gamma}(t)=\omega\right)=\frac{q(\omega)}{Z_{n}}, \quad \text { for all } \omega \in \Omega_{n}^{0},
$$

and try to compute $\operatorname{Prob}\left(X_{\alpha \beta \gamma}(t+1)=\omega^{\prime}\right)$.

Recall that $I(t)$ denotes the wall chosen at time $t$, so that $X_{\alpha \beta \gamma}(t+1)=$ $T\left(X_{\alpha \beta \gamma}(t), I(t)\right)$, and define $J(t+1)$ by $\bar{T}\left(X_{\alpha \beta \gamma}(t), I(t)\right)=\left(X_{\alpha \beta \gamma}(t+1), J(t+1)\right)$ if $I(t)$ became active, or by $J(t+1)=I(t)$ otherwise. Then, in view of the first point in Theorem 1.2,

$$
\operatorname{Prob}\left(X_{\alpha \beta \gamma}(t+1)=\omega^{\prime}\right)=\sum_{j=0}^{n} \operatorname{Prob}\left(X_{\alpha \beta \gamma}(t+1)=\omega^{\prime}, J(t+1)=j\right) .
$$

Now, by definition of the Markov chain $X_{\alpha \beta \gamma}$, for all $\omega^{\prime}$ and $j$,

$$
\begin{aligned}
\operatorname{Prob}\left(X_{\alpha \beta \gamma}(t+1)=\omega^{\prime},\right. & J(t+1)=j) \\
= & \lambda(i) \cdot \operatorname{Prob}\left(X_{\alpha \beta \gamma}(t)=\omega, I(t)=i\right) \\
& +(1-\lambda(j)) \cdot \operatorname{Prob}\left(X_{\alpha \beta \gamma}(t)=\omega^{\prime}, I(t)=j\right),
\end{aligned}
$$

where $(\omega, i)=\bar{T}^{-1}\left(\omega^{\prime}, j\right)$. Since the random variable $I(t)$ is uniform on $\{0, \ldots, n\}$, we get

$$
\begin{aligned}
\operatorname{Prob}\left(X_{\alpha \beta \gamma}(t+1)=\omega^{\prime},\right. & J(t+1)=j) \\
& =\lambda(i) \cdot \frac{q(\omega)}{Z_{n}} \frac{1}{n+1}+(1-\lambda(j)) \cdot \frac{q\left(\omega^{\prime}\right)}{Z_{n}} \frac{1}{n+1} .
\end{aligned}
$$

But according to the second point in Theorem 1.2, $\lambda(i) q(\omega)=\lambda(j) q\left(\omega^{\prime}\right)$ so that the terms involving $\lambda$ cancel. Finally

$$
\operatorname{Prob}\left(X_{\alpha \beta \gamma}(t+1)=\omega^{\prime}\right)=\sum_{j=0}^{n} \frac{q\left(\omega^{\prime}\right)}{Z_{n}} \frac{1}{n+1}=\frac{q\left(\omega^{\prime}\right)}{Z_{n}},
$$

and this completes the proof that the distribution induced by $q$ is stationary. 


\subsection{From the complete to the basic system.}

The relation $S_{\alpha \beta \gamma} \equiv \operatorname{top}\left(X_{\alpha \beta \gamma}\right)$ now allows to derive from Theorem 1.1 a combinatorial interpretation for the basic system.

Theorem 1.3 Let top $(\omega)$ denote the top row of a complete configuration $\omega$. Then for any initial configurations $S_{\alpha \beta \gamma}(0)$ and $X_{\alpha \beta \gamma}(0)$ with $t o p\left(X_{\alpha \beta \gamma}(0)\right)=S_{\alpha \beta \gamma}(0)$, and any basic configuration $r$,

$$
\operatorname{Prob}\left(S_{\alpha \beta \gamma}(t)=r\right)=\operatorname{Prob}\left(t o p\left(X_{\alpha \beta \gamma}(t)\right)=r\right) \underset{t \rightarrow \infty}{\longrightarrow} \frac{1}{Z_{n}} \sum_{\left\{\omega \in \Omega_{n}^{0} \mid t o p(\omega)=r\right\}} q(\omega) .
$$

In particular, in the case $\alpha=\beta=\gamma=1$, we recover

$$
\operatorname{Prob}\left(S_{\alpha \beta \gamma}(t)=r\right) \underset{t \rightarrow \infty}{\longrightarrow} \frac{\left|\left\{\omega \in \Omega_{n}^{0} \mid t o p(\omega)=r\right\}\right|}{\left|\Omega_{n}^{0}\right|},
$$

which is yields

$$
\operatorname{Prob}\left(S_{\alpha \beta \gamma}(t) \text { contains } k \text { black particles }\right) \underset{t \rightarrow \infty}{\longrightarrow} \frac{\left|\Omega_{k, m}^{0}\right|}{\left|\Omega_{n}^{0}\right|}=\frac{\frac{1}{n+1}\left(\begin{array}{c}
n+1 \\
k
\end{array}\right)\left(\begin{array}{c}
n+1 \\
m
\end{array}\right)}{C_{n+1}} .
$$

As discussed in Section 4 this interpretation sheds a new light on some recent results of Derrida et al. connecting the TASEP to Brownian excursions [2].

\subsection{Continuous-time descriptions of the TASEP}

In the physics literature, the TASEP is usually described in the following terms. The time is continuous, and one consider walls where a move can take place: at any time, wall $i$ has probability $\lambda(i) d t$ to trigger a move $\omega \rightarrow \vartheta(\omega, i)$ during time interval $d t$ (the rate $\lambda(i)$ is defined as previously).

Following the probabilistic literature [7], one can give an formulation which is equivalent to the previous one, but already closer to ours. In this description, each wall waits for an independent exponential random time with rate 1 before waking up (in other terms, the probability that wall $i$ will still be sleeping in $t$ seconds is $\left.e^{-t}\right)$. When wall $i$ wakes up, it has probability $\lambda(i)$ to become active. If this is the case, then the transition $\omega \rightarrow \vartheta(\omega, i)$ is applied to the current configuration $\omega$. In any case the wall falls again asleep, restarting its clock again.

This continuous-time TASEP is now easily coupled to the Markov chain $S_{\alpha \beta \gamma}$. Let the time steps of $S_{\alpha \beta \gamma}$ correspond to the succession of moments at which a wall wakes up. Then in both versions, the index of next wall to wake up is at any time an uniform random variable on $\{0, \ldots, n\}$, and when a wall wakes up the transition probabilities are identical. This implies that the stationary distribution of the continuous-time TASEP and its Markov chain replica are identical.

\subsection{Outline of the rest of the paper.}

In Section 2 an approach to compute explicit quantities from the combinatorial interpretation is briefly exposed. Theorem 1.2 is proved in Section 3. Finally some concluding remarks are gathered in Section 4. 


\section{Enumeration}

Let us introduce the weighted generating function of complete configurations with respect to their length:

$$
Z(t ; u, v)=\sum_{n \geq 0} \sum_{\omega \in \Omega_{n}^{0}} u^{n_{x}(\omega)} v^{n_{y}(\omega)} t^{n}, \quad \text { so that } \quad Z\left(\beta \gamma t ; \frac{\alpha}{\gamma}, \frac{\alpha}{\beta}\right)=\sum_{n \geq 0} Z_{n} t^{n} .
$$

The decomposition of a configuration at its first block yields

$$
Z(t ; u, v)=1+t v Z(t ; u, v)+t u Z(t ; 1, v)+t^{2} v Z(t ; 1,1) Z(t ; u, v) .
$$

Solving this equation yields

$$
Z(t ; u, v)=\frac{2-u-v+u v-2 t u v-(u+v-u v) \sqrt{1-4 t}}{2\left(1-u+t u^{2}\right)\left(1-v+t v^{2}\right)} .
$$

Extracting coefficients in this expression allow to recover for instance a formula for $Z_{n}$. One could also have taken into account the number of particles in the top row in the equation. We do not pursue on this line since $Z_{n}$ was obtained by other ways and largely studied as a function of $\alpha, \beta, \gamma$ in the physical literature.

\section{The bijection $\bar{T}$}

The aim of this section is to prove Theorem 1.2, thus giving an alternate description of the transformation $T$ and a case by case analysis of its action on the weight $q$. We need the following properties, the verification of which is left to the reader.

Property 3.1 Let $\omega$ be a complete configuration belonging to $\Omega_{n}$, then we have the following structure properties:

1. In a local configuration $\left.\left.\right|_{?} ^{\bullet}\right|_{\bullet} ^{0} \mid$ the black particle in the bottom row never contributes a label $y$.

2. The white particle in the bottom row of a $\left.\right|_{0} ^{\circ} \mid$-column never has a label $x$.

and movement properties:

$i$. The deletion/insertion of a $\left.\right|_{.} ^{\circ}$ - -column does not change the labels of other particles.

ii. The deletion/insertion of a pair $\left.\bullet\right|_{0}$ taking the form $\left|\begin{array}{l}\bullet \\ ?\end{array}\right| \begin{aligned} & 0 \\ & 0\end{aligned}|\leftrightarrow| \begin{aligned} & 0 \\ & ?\end{aligned} \mid$ does not change the labels of other particles.

From now on in this section, $(\omega, i)$ denotes an element of the current class, and $\left(\omega^{\prime}, j\right)$ its image by $\bar{T}$. In the pairs $(\omega, i)$ and $\left(\omega^{\prime}, j\right), i$ and $j$ refer to walls of the configurations $\omega$ and $\omega^{\prime}$, and $i$ is called the active wall of $\omega$. Following the notations of Section 1 , when $i \neq 0$, we also consider $j_{1}<i$ the smallest integer such that in the top row of $\omega$ all cells between walls $j_{1}$ and $i-1$ contain white particles. Symmetrically, when $i \neq n$, we consider $j_{2}>i$ the largest integer such that in the top row of $\omega$ all cells between walls $i+1$ and $j_{2}$ contain black particles. 

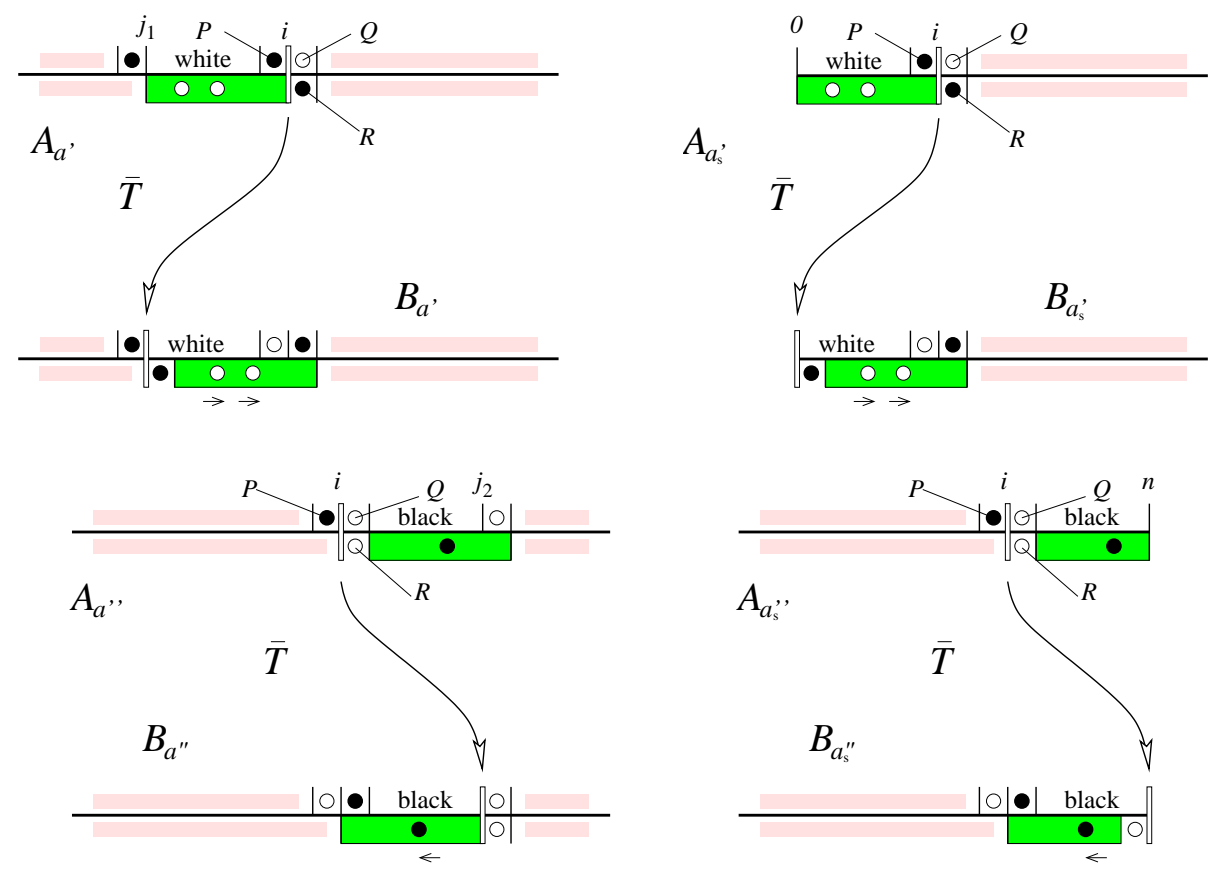

Figure 8: Jump moves in the case $\bullet \mid \circ \rightarrow \circ$

To define the bijection $\bar{T}$ and prove Theorem 1.2 we shall partition the set $\Omega_{n} \times\{0, \ldots, n\}$ into classes $A_{a^{\prime}}, A_{a_{s}^{\prime}}, A_{a^{\prime \prime}}, A_{a_{s}^{\prime \prime}}, A_{b}, A_{b_{s}}, A_{c}, A_{c_{s}}, A_{d}$, and describe, for each class $A_{z}$, its image $B_{z}=\bar{T}\left(A_{z}\right)$ under the action of $\bar{T}$ and the corresponding variation of the weight $q$ :

The active wall of $\omega$ separates in the top row a black particle $P$ and a white particle $Q$. Then in the top row the particles $P$ and $Q$ swap. In the bottom row, the sweep that occurs depends on the type of the particle $R$ that is below $Q$ in $\omega$ (see Figure 8):

$A_{a^{\prime}}$ The particle $R$ is black and the wall $j_{1}$ is different from 0 . Then $j=j_{1}$ and, in the bottom row, a white sweep occurs between walls $j$ and $i$. The new configuration $\omega^{\prime}$ belongs to $\Omega_{n}$. Indeed $\omega^{\prime}$ can also be described as obtained from $\omega$ by moving a $\left.\right|_{0} ^{0} \mid$-column from the right of the $i$ th wall to the right of the $j_{1}$ th. But moving a $\left.\right|_{\bullet} ^{0} \mid$-column has no effect on the positivity constraint.

Now we want to compare $q(\omega)$ and $q\left(\omega^{\prime}\right)$. According to Property 3.1.1 the particle $R$ does not contribute a label $y$ neither in $\omega$ nor in $\omega^{\prime}$. Moreover, according to Property 3.1.i, the displacement of the $\left.\right|_{.} ^{\circ} \mid$-column does not affect labels of other particles. Hence $q(\omega)=q\left(\omega^{\prime}\right)$, in agreement with $\lambda(i)=$ $\lambda(j)$.

The image $B_{a^{\prime}}$ of the class $A_{a^{\prime}}$ consists of pairs $\left(\omega^{\prime}, j\right), j>0$, with a $\left.\right|^{\circ}$. column on the right hand side of the $j$ th wall of $\omega^{\prime}$ and such that the sequence of white particles on the right hand side of the $j$ th wall in the top row is followed by a black particle. 
$A_{a_{s}^{\prime}}$ The particle $R$ is black and the wall $j_{1}=0$. Then $j=0$ and a white sweep occurs between walls 0 and $i$. The new configuration $\omega^{\prime}$ still belongs to $\Omega_{n}$ since it is again obtained from $\omega$ by moving a $\left.\right|_{.} ^{\circ}$--column from the right of the $i$ th wall to the right of the wall 0 .

As opposed to the previous case, Property 3.1.1 applies only to $\omega$ : in $\omega^{\prime}$, the displaced $\left.\right|_{0} ^{\circ} \mid$-column is the leftmost one, so that its black particle contributes a supplementary $y$ label. Therefore $q\left(\omega^{\prime}\right)=q(\omega) \frac{\alpha}{\beta}$, in agreement with $\lambda(i)=$ $\alpha, \lambda(0)=\beta$.

The image $B_{a_{s}^{\prime}}$ consists of pairs $\left(\omega^{\prime}, 0\right)$ such that the top row starts on the left by a non-empty sequence of white particles followed by a black one.

$A_{a^{\prime \prime}}$ The particle $R$ is white and the wall $j_{2}$ is different from $n$. Then $j=j_{2}$ and, in the bottom row, a black sweep occurs between walls $i+1$ and $j+1$. The new configuration $\omega^{\prime}$ belongs to $\Omega_{n}$. Indeed $\omega^{\prime}$ can be described as obtained from $\omega$ by moving a ${ }^{\bullet}$-diagonal from the $i$ th wall to the $j_{2}$ th wall: this movement has no effect on the positivity constraint.

From Property 3.1.2 we see that the particle $R$ does not contribute a label $y$ neither in $\omega$ nor in $\omega^{\prime}$, and from Property 3.1.ii the displacement of the $\bullet$ o-diagonal does not affect labels of other particles. Hence $q(\omega)=q\left(\omega^{\prime}\right)$, in agreement with $\lambda(i)=\lambda(j)$.

The image $B_{a^{\prime \prime}}$ of the class $A_{a^{\prime \prime}}$ consists of pairs $\left(\omega^{\prime}, j\right)$ with a $\left.\right|_{\circ} ^{\circ} \mid$-column on the right hand side of the $j$ th wall and such that the sequence of black particles on the left hand side of the $j$ th wall in the top row is followed by a white particle.

$A_{a_{s}^{\prime \prime}}$ The particle $R$ is white and the wall $j_{2}$ is equal to $n$. Then $j=n$ and, in the bottom row, a black sweep occurs between walls $i+1$ and $n$. The new configuration $\omega^{\prime}$ still belongs to $\Omega_{n}$ since it is obtained from $\omega$ by removing $\left.\mathrm{a} \cdot\right|_{0}$-diagonal around the $i$ th wall and inserting a $\left.\right|_{0} ^{\bullet} \mid$-column to the left of the $n$-th wall.

As opposed to the previous case, Property 3.1.2 applies only to $\omega$ : the inserted |:-column is the rightmost one, so that its white particle contributes an $x$ label. Therefore $q\left(\omega^{\prime}\right)=q(\omega) \frac{\alpha}{\gamma}$, in agreement with $\lambda(i)=\alpha$ and $\lambda(n)=\gamma$.

The image $B_{a_{s}^{\prime \prime}}$ of the class $A_{a_{s}^{\prime \prime}}$ consists of pairs $\left(\omega^{\prime}, n\right)$ such that there is a sequence of black particles on the left hand side of the $n$th wall in the top row, followed by a white particle.

The active wall of $\omega$ is the left border with a white particle $Q$ on its right in the top row. Again, the cell under $Q$ must contain a black particle $R$ (see Figure 9 ). First the two particle $Q$ and $R$ exchange to form a $\left.\right|_{0} ^{\bullet} \mid$-column. Then we have two cases:

$A_{b}$ the wall $j_{2}$ is not $n$. Then $j=j_{2}$ and, in the bottom row, a black sweep occurs between walls 1 and $j+1$. The configuration $\omega^{\prime}$ belongs to $\Omega_{n}$. Indeed no black particle moves to the right.

Equivalently, the new configuration $\omega^{\prime}$ is obtained by inserting a $\left.\bullet\right|_{0}$-diagonal at the wall $j_{2}$ and deleting the first $\left.\right|^{\circ}$. -column. According to Properties 3.1.i and 3.1.ii only the labels of the displaced particles are affected. Since the deleted $\left.\right|_{0} ^{\circ} \mid$-column is the leftmost, it contributes a $y$ label in $\omega$. As opposed 

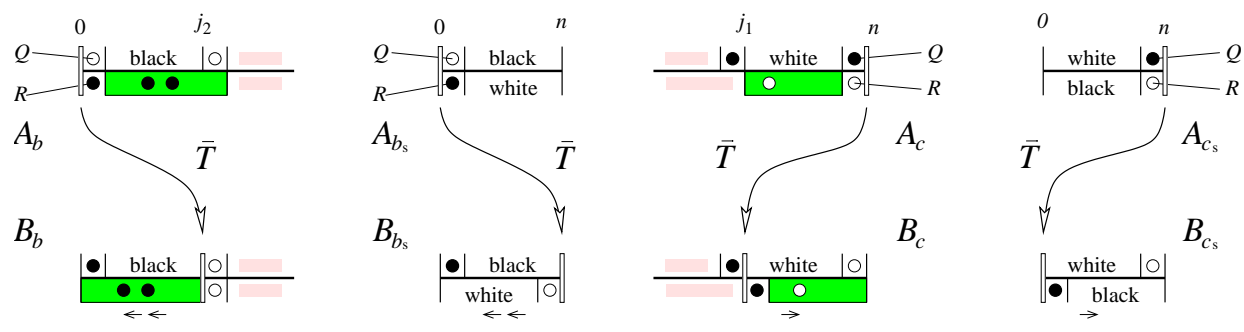

Figure 9: Active left border and active right border with respectively a black and a white particle in the top row.

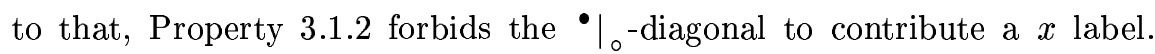
Therefore $q\left(\omega^{\prime}\right)=q(\omega) \frac{\beta}{\alpha}$, in agreement with $\lambda(0)=\beta$ and $\lambda(j)=\alpha$.

The image $B_{b}$ consist of pairs $\left(\omega^{\prime}, j\right)$ with a $\left.\right|_{0} ^{0} \mid$-column on the right of the $j$ th wall of $\omega^{\prime}$ and such that the sequence of black particles on left of the $j$ th wall in the top row ends at the border.

$A_{b_{s}}$ the wall $j_{2}$ is equal to $n$. Then $j=n$ and, in the bottom row, a black sweep occurs between walls 1 and $n$. Finally, $q\left(\omega^{\prime}\right)=q(\omega) \frac{\beta}{\alpha} \frac{\alpha}{\gamma}=q(\omega) \frac{\beta}{\gamma}$, in agreement with $\lambda(0)=\beta$ and $\lambda(n)=\gamma$.

The image $B_{b_{s}}$ is reduced to the configuration with all black particles in the top row.

The active wall of $\omega$ is the right border with a black particle $Q$ on its left in the top row. The cell under $Q$ must contain a white particle $R$ (see Figure 9): First the particles $Q$ and $R$ exchange to form a $\left.\right|_{\bullet} ^{\circ} \mid$-column. There are then two cases:

$A_{c}$ the wall $j_{1}$ is different from 0 . Then $j=j_{1}$ and in the bottom row, a white sweep occurs between walls $j$ and $n-1$. The configuration $\omega^{\prime}$ belongs to $\Omega_{n}$ since the transformation amounts to moving and flipping a $\left.\right|_{0} ^{\bullet} \mid$-column.

Equivalently, $\omega^{\prime}$ is obtained by inserting a $\left.\right|_{0} ^{0} \mid$-column at the left of the wall $n$ and deleting the $\left.\right|_{0} ^{\circ} \mid$-column on the right of $j_{1}$. According to Property 3.1.i only the labels of displaced particles can be affected. Since the deleted $|\bullet|-$ column is the rightmost column, its white particle contributes an $x$ label in $\omega$. As opposed to that, Property 3.1.1 forbids the $\left.\right|_{0} ^{\circ} \mid$-column to contribute a label in $\omega^{\prime}$. Therefore $q\left(\omega^{\prime}\right)=q(\omega) \frac{\gamma}{\alpha}$, in agreement with $\lambda(n)=\gamma$ and $\lambda(j)=\alpha$.

The image $B_{c}$ consist of pairs $\left(\omega^{\prime}, j\right)$ with a $\left.\right|_{.} ^{\circ} \mid$-column on the right hand side of the $j$ th wall of $\omega^{\prime}$ and such that the sequence of white particles on the right hand side of the $j$ th wall in the top row ends at the right border.

$A_{c_{s}}$ the wall $j_{1}$ is equal to 0 . Then $j=0$ and in the bottom row, a white sweep occurs between walls 0 and $n-1$. Finally, $q\left(\omega^{\prime}\right)=q(\omega) \frac{\gamma}{\alpha} \frac{\alpha}{\beta}=q(\omega) \frac{\gamma}{\beta}$, in agreement with $\lambda(n)=\gamma$ and $\lambda(0)=\beta$.

The image $B_{b_{s}}$ is reduced to the configuration with all white particles in the top row. 
$A_{d}$ : This class contains all the remaining cases. On these pairs the application $\bar{T}$ has no effect, that is, for $(\omega, i) \in A_{d}, \bar{T}(\omega, i)=(\omega, i)$. In particular the weights are left unchanged.

The observation that image classes $\left\{B_{a^{\prime}}, B_{a_{s}^{\prime}}, B_{a^{\prime \prime}}, B_{a_{s}^{\prime \prime}}, B_{b}, B_{b_{s}}, B_{c}, B_{c_{s}}, B_{d}\right\}$ form a partition of $\Omega_{n} \times\{0, \ldots, n\}$ completes the proof of Theorem 1.2.

\section{Conclusions and links to Brownian excursions}

The starting point of the paper [5] was a "combinatorial ansatz": the stationary distribution of the TASEP can be expressed in terms of Catalan numbers hence should have a nice combinatorial interpretation. As we have seen in the present paper, our approach is natural enough to extend to more general TASEP.

We do not claim that our combinatorial interpretation is of any physical relevance. However, as already pointed out in [5], apart from explaining the occurrence of "magical" Catalan numbers in the problem, it sheds a new light on the recent results of Derrida et al. [2] connecting the TASEP with Brownian excursion. More precisely, using explicit calculations, Derrida et al. show that when $\alpha=\beta=\gamma=1$, the density of black particles in configurations of the TASEP can be expressed in terms of a pair $\left(e_{t}, b_{t}\right)$ of independent processes, a Brownian excursion $e_{t}$ and a Brownian motion $b_{t}$. In our interpretation these two quantities appear at the discrete level, associated to each complete configuration $\omega$ of $\Omega_{n}^{0}$ :

- The role of the Brownian excursion is played for $\omega$ by the halved differences $e(i)=\frac{1}{2}(B(i)-W(i))$ between the number of black and white particles sitting on the left of the $i$ th wall, for $i=0, \ldots, n$. By definition of complete configurations, $(e(i))_{i=0, \ldots, n}$ is a discrete excursion, that is, $e(0)=e(n)=0$, $e(i) \geq 0$ and $|e(i)-e(i-1)| \in\{0,1\}$, for $i=0, \ldots, n$.

- The role of the Brownian motion is played for $\omega$ by the differences $b(i)=$ $B_{t o p}(i)-B_{b o t}(i)$ between the number of black particles sitting in the top and in the bottom row, on the left of the $i$ th wall, for $i=0, \ldots, n$. This quantity $(b(i))_{i=0, \ldots, n}$ is a discrete walk, with $|b(i)-b(i-1)| \in\{0,1\}$ for $i=0, \ldots, n$.

Since $e(i)+b(i)=2 B_{t o p}(i)-i$, these quantities allow to describe the cumulated number of black particles in the top row of a complete configuration. Accordingly, the density in a given segment $(i, j)$ is

$$
\frac{B_{t o p}(j)-B_{t o p}(i)}{j-i}=\frac{1}{2}+\frac{e(j)-e(i)}{2(j-i)}+\frac{b(j)-b(i)}{2(j-i)} .
$$

This is a discrete version of the quantity considered by Derrida et al. in [2].

Now the two walks $e(i)$ and $b(i)$ are correlated since one is stationary when the other is not, and vice versa: $|e(i)-e(i-1)|+|b(i)-b(i-1)|=1$. Given $\omega$, let $I_{e}=\left\{\alpha_{1}<\ldots<\alpha_{p}\right\}$ be the set of indices of $|:|-$ and $\left.\right|_{0} ^{0} \mid$-columns, and $I_{b}=\left\{\beta_{1}<\ldots<\beta_{q}\right\}$ the set of indices of $\left.\right|_{0} ^{\bullet} \mid-$ and $\left.\right|_{0} ^{0} \mid$-columns $(p+q=n)$. Then the walk $e^{\prime}(i)=e\left(\alpha_{i}\right)-e\left(\alpha_{i-1}\right)$ is the excursion obtained from $e$ by ignoring stationary steps, and the walk $b^{\prime}(i)=b\left(\beta_{i}\right)-b\left(\beta_{i-1}\right)$ is obtained from $b$ in the same way. Conversely given a simple excursion $e^{\prime}$ of length $p$, a simple walk $b^{\prime}$ of length $q$ and a subset $I_{e}$ of $\{1, \ldots, p+q\}$ of cardinal $p$, two correlated walks $e$ and $b$, and thus a complete configuration $\omega$ can be uniquely reconstructed. The consequence of this discussion is that the uniform distribution on $\Omega_{n}^{0}$ (which is 
stationary for $\alpha=\beta=\gamma=1$ ) corresponds to the uniform distribution of triples $\left(I_{e}, e^{\prime}, b^{\prime}\right)$ where given $I_{e}, e^{\prime}$ and $b^{\prime}$ are independent.

A direct computation shows that in the large $n$ limit, with probability exponentially close to 1 , a random configuration $\omega$ is described by a pair $\left(e^{\prime}, b^{\prime}\right)$ of walks of roughly equal lengths $n / 2+O\left(n^{1 / 2+\varepsilon}\right)$. In particular up to multiplicative

constants the normalized pairs $\left(\frac{e^{\prime}(t n / 2)}{n^{1 / 2}}, \frac{b^{\prime}(t n / 2)}{n^{1 / 2}}\right)$ and $\left(\frac{e(t n)}{n^{1 / 2}}, \frac{b(t n)}{n^{1 / 2}}\right)$ both converge to the same pair $\left(e_{t}, b_{t}\right)$ of independent processes, with $e_{t}$ a standard Brownian excursion and $b_{t}$ a standard Brownian walk.

We thus obtained a combinatorial interpretation of the apparition of the pair $\left(e_{t}, b_{t}\right)$ in the TASEP at $\alpha=\beta=\gamma=1$. How do these considerations extend to other TASEP? The case $\alpha$ much smaller than $\beta$ and $\gamma$ essentially reduces to $\alpha=\beta=\gamma=1$ on a system of length $n-2$ (with border cells acting as reservoirs). The case $\beta$ and $\gamma$ smaller than $\alpha$ appears more interesting to consider: at a rough level, the weights force $e(t)$ to spend more time at the value exactly zero and favors negative value of $b(t)$. The derivation of the associated continuum quantities in this case could be of some interest.

Another challenge raised by our approach is to give an explicit construction of a continuum TASEP by taking the limit of the Markov chain $X_{\alpha \beta \gamma}$, viewed as a Markov chain on pairs of walks. An appealing way to give a geometric meaning to the transitions in the continuum limit could be to use a representation in terms of parallelogram polyominos, where the process $e(t)$ (or $e_{t}$ in the continuum limit) describes the width of the polymonino and $b(t)$ (or $b_{t}$ in the continuum limit) describes its vertical displacement.

\section{References}

[1] B. Derrida, E. Domany, and D. Mukamel. An exact solution of a one dimensional asymmetric exclusion model with open boundaries. J. Stat. Phys., 69:667-687, 1992.

[2] B. Derrida, C. Enaud, and J. L. Lebowitz. The asymmetric exclusion process and brownian excursions. Available electronically as arXiv: cond-mat/0306078.

[3] B. Derrida, M.R. Evans, V. Hakim, and V. Pasquier. Exact solution of a one-dimensional asymmetric exclusion model using a matrix formulation. $J$. Phys. A: Math., 26:1493-, 1993.

[4] B. Derrida, J. L. Lebowitz, and E. R. Speer. Exact large deviation functional of a stationary open driven diffusive system: the asymmetric exclusion process. Available electronically as arXiv: cond-mat/0205353.

[5] E. Duchi and G. Schaeffer. A combinatorial approach to jumping particles I: maximal flow regime. In proceedings of FPSAC'04, 2004.

[6] O. Häggström. Finite Markov Chains and Algorithmic Applications. Cambridge University Press, 2002.

[7] T. M. Liggett. Interacting Particle Systems. Springer, New York, 1985.

[8] R. Stanley. Enumerative Combinatorics, volume II. Cambridge University Press, 1999. 


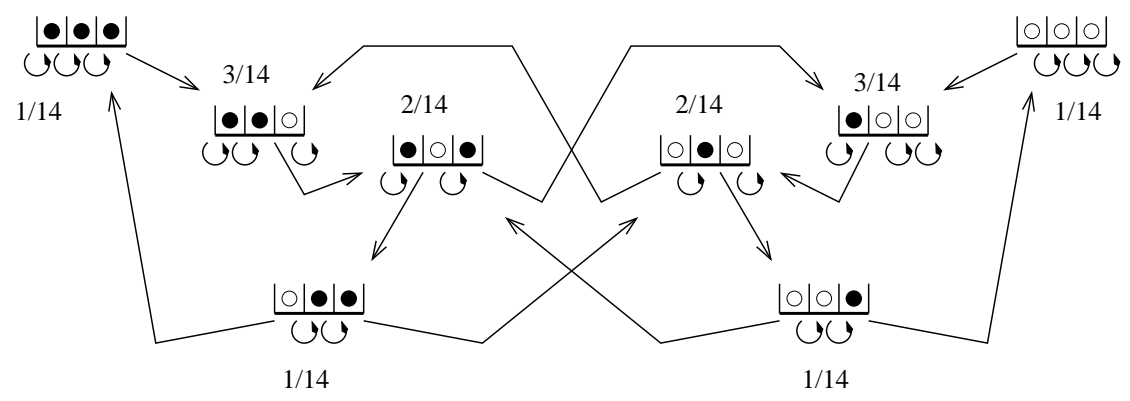

Figure 10: The basic configurations for $n=3$ and transitions between them. The startpoint of each arrow indicates the wall triggering the transition. The numbers are the stationary probabilities.

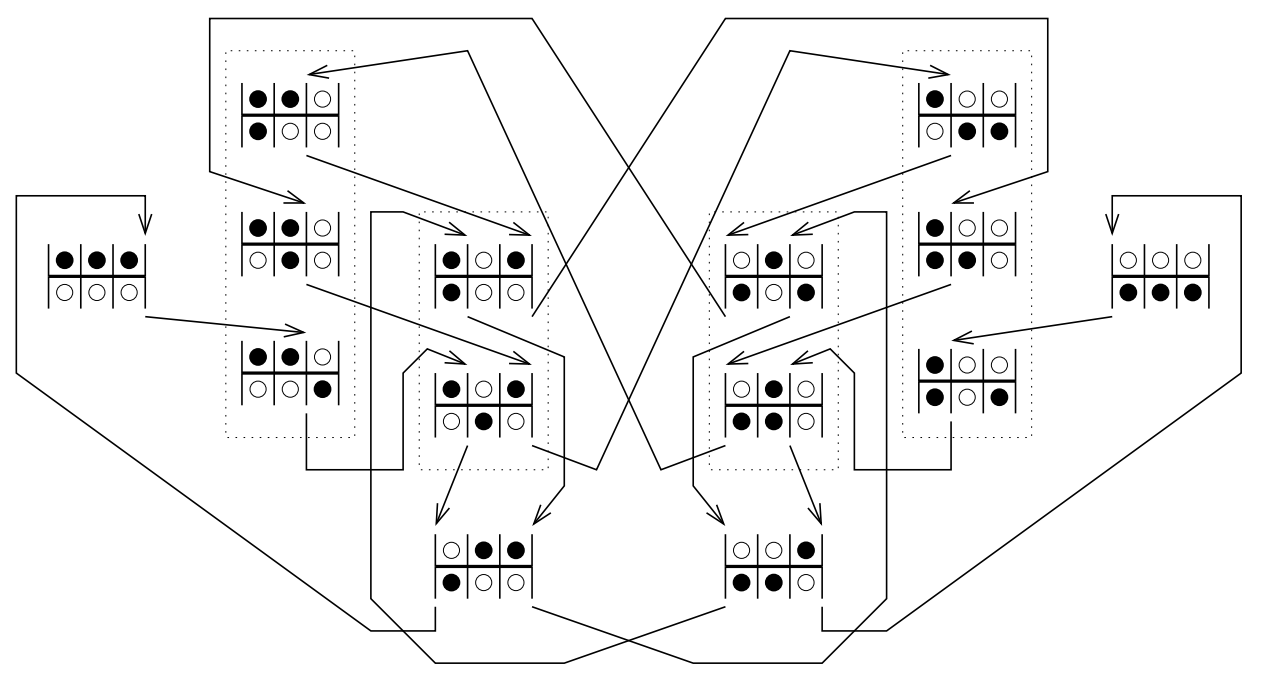

Figure 11: The 14 complete configurations for $n=3$ and transitions between them. The startpoint of each arrow indicates the wall triggering the transition (loop transitions are not indicated). Stationary probabilities are uniform (equal to $1 / 14$ ) since each configuration has equal in and out degrees. Ignoring bottom rows reduces this Markov chain to the chain of Figure 10.

Enrica Duchi

CAMS, EHESS, 87, bd Raspail, 75006 Paris, France

Enrica.Duchi@ehess.fr

Gilles Schaeffer

LIX, CNRS - École Polytechnique, 91128 Palaiseau, France

Gilles.Schaeffer@lix.polytechnique.fr 